\title{
Surgical Management of Dysplasia and Cancer in Inflammatory Bowel Disease
}

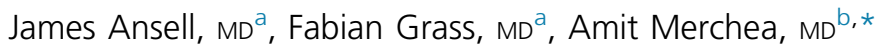

\section{KEYWORDS}

- Crohn • Ulcerative colitis • Cancer • Dysplasia

\section{KEY POINTS}

- Patients with inflammatory bowel disease (IBD) and high-grade dysplasia, multifocal lowgrade dysplasia and nonadenoma dysplasia-associated mass or lesion, high-grade or invasive cancer should undergo total proctocolectomy.

- Segmental resection in IBD-colorectal cancer (CRC) has a limited role because of the risk of developing secondary malignancies in other areas of colon.

- Ileal pouch anal anastomosis (IPAA) surgery for ulcerative colitis restores intestinal continuity and is associated with a very small risk of pouch neoplasia. High-risk patients with a history of dysplasia or CRC before IPAA should undergo pouchoscopy with biopsy every 1 to 3 years.

- IPAA surgery should not be considered in patients with a history of pelvic radiation, and indications for pouch excision include biopsy-confirmed high-grade dysplasia or synchronous cancer.

\section{INTRODUCTION}

Chronic inflammatory bowel disease (IBD) is characterized by recurrent episodes of mucosal inflammation, which can lead to an increased risk of dysplasia and cancer. ${ }^{1}$ It is nearly a century since Crohn and Rosenberg ${ }^{2}$ published their data linking IBD with colorectal cancer (CRC). A 2001 meta-analysis reported the overall IBD-CRC risk was 3 per 1000 per year and $2 \%, 8 \%$ and $18 \%$ in the first, second, and third decades after diagnosis, respectively. ${ }^{3}$ More than a decade later, this risk has decreased to 1.69 per 1000 per year, possibly because of improved early detection and surveillance, earlier

Disclosure: The authors declare no relationship with a commercial company that has a direct financial interest in subject matter or materials discussed in the article or with a company making a competing product.

a Division of Colon and Rectal Surgery, Mayo Clinic, 200 First Street Southwest, Rochester, MN 55905, USA; b Division of Colon and Rectal Surgery, Mayo Clinic, 4500 San Pablo Road South, Jacksonville, FL 32224, USA

* Corresponding author.

E-mail address: Merchea.Amit@mayo.edu 
surgical intervention in medically refractory disease, and the introduction of chemoprotective aminosalicytes. ${ }^{4,5}$

The overall incidence of IBD-CRC is $2.5 \%$, but this varies based on the site of inflammation. ${ }^{4,6}$ Colonic Crohn's disease (CD) is associated with CRC (relative risk, 4.5 [1.3-14.9]), but ileal CD is not (relative risk, $1.1[0.8-1.5])^{4,6}$ The cumulative risk of $\mathrm{CRC}$ in $\mathrm{CD}$ at 10,20 , and 30 years after diagnosis is $2.9 \%, 5.6 \%$, and $8.3 \%$, respectively.

The cause and presentation of IBD-CRC differs from that of sporadic CRC. Sporadic disease is linked to the predictable adenoma-carcinoma sequence, but IBD$\mathrm{CRC}$ is driven by cellular damage from chronic inflammation and does not follow in such an orderly fashion. ${ }^{7,8}$ IBD-CRC tends to present at a younger age compared with sporadic disease (mean, $40-50$ years vs 60 years) ${ }^{9}$ and may arise in flat mucosa that is more difficult to visualize. ${ }^{10}$

Risk factors for IBD-CRC include a younger age at onset, duration greater than 8 years, extent of colitis, and the degree of endoscopic and histologic inflammation. ${ }^{11,12}$ Other risks include primary sclerosing cholangitis (PSC) (odds ratio, 4.8; $95 \%$ confidence interval $[\mathrm{Cl}], 3.6-6.4)^{13}$ and a positive family history of CRC. ${ }^{14}$

Previous work by our group detailed the risk, pathogenesis, surveillance, and management of IBD dysplasia and CRC. ${ }^{15}$ This article focuses on the surgical approach to IBD dysplasia and CRC.

\section{COLORECTAL CANCER SCREENING}

Current data on the survival benefit for screening in IBD are sparse, with a Cochrane Review failing to show a benefit, ${ }^{16}$ so our review focuses on expert panel consensus. ${ }^{12}$

Most current guidelines recommend starting surveillance colonoscopy 6 to 10 years after the diagnosis of IBD, with surveillance colonoscopy every 1 to 2 years. ${ }^{17-21}$ In the presence of coexisting PSC, patients should undergo colonoscopy followed by yearly surveillance. ${ }^{17}$ Of note, PSC may be an indication for prophylactic colectomy, although the relative risk of IBD-CRC is lower in CD than in ulcerative colitis (UC). ${ }^{22}$

Surveillance technique normally involves 2 sets of 4-quadrant biopsies in each colon segment (right, transverse, left, rectosigmoid), producing more than 32 random biopsies, providing an $80 \%$ to $90 \%$ sensitivity for dysplasia. ${ }^{23-25}$ Directed biopsies of polypoid lesions, masses, strictures, or irregular mucosa should also be performed. ${ }^{23,26,27}$

Recent research has shown that dysplasia may be better identified with advanced endoscopic imaging and targeted biopsies rather than random biopsies. ${ }^{28-30}$

\section{Advanced Endoscopic Imaging}

Chromoendoscopy (CE) uses a dye agent such as indigo carmine or methylene blue to enhance the detection of mucosal lesions. ${ }^{30,31}$ This technique improves flat dysplastic lesion detection by $27 \% .{ }^{32,33}$ At present, most expert societies recommend the use of CE with targeted biopsies whenever the technology and expertise are available, particularly for high-risk patients. ${ }^{33}$

Digital or optical imaging technology, such as narrow band imaging (NBI; Olympus, Tokyo, Japan), uses specific light wavelengths that penetrate only the superficial mucosa to better delineate mucosal vasculature without the use of dyes, ${ }^{34,35}$ but this has not been shown to provide a benefit in neoplasia detection rates. ${ }^{36}$ Compared with chromoendoscopy, NBI is associated with a higher miss rate for neoplasia, and is not recommended for IBD surveillance. ${ }^{35}$ 
High-resolution endoscopy and endocytoscopy are emerging areas of interest for endoscopic surveillance, but have yet to show improved detection of neoplasia compared with conventional endoscopy. ${ }^{37-43}$

\section{MANAGEMENT OF INFLAMMATORY BOWEL DISEASE-RELATED DYSPLASIA, CANCER, AND STRICTURES}

Dysplasia can be microscopically classified into low-grade dysplasia (LGD), highgrade dysplasia (HGD), or indefinite for dysplasia depending on the degree of preservation or loss of cellular polarity reflected by nuclear stratification. ${ }^{44}$ Dysplasia identified on random biopsies must be confirmed by 2 gastrointestinal pathologists. One of the most important determinants in managing patients with raised dysplastic lesions is whether the dysplasia is completely resectable. ${ }^{44}$ If a lesion is nonresectable because of size, fixation, or adjacent dysplasia, it is referred to as a dysplasiaassociated lesion or mass (DALM). DALMs can be further divided into adenomalike (polypoid) and nonadenomalike (nonpolypoid). ${ }^{45}$

Adenomalike DALMs tend to behave like sporadic adenomas and can be safely treated with polypectomy, tattoo, and continued surveillance, ${ }^{46}$ a strategy associated with a low risk of progression to CRC $(2.4 \%$ of patients after an average follow-up of 54 months). ${ }^{47}$ However, if biopsy of the surrounding tissue identifies dysplasia, colectomy is recommended because of the high risk of associated CRC. ${ }^{10,29,47}$

Nonadenomalike DALMs can appear as velvety patches, plaques, irregular bumps and nodules, wartlike thickenings, stricturing lesions, or broad-based masses. ${ }^{45}$ These flat DALMs are usually not amenable to endoscopic removal, and they have a 9-fold risk of developing cancer and a 12-fold risk of developing any advanced lesion, ${ }^{48}$ so surgical resection is usually warranted.

\section{Low-Grade Dysplasia}

The management of unifocal, flat LGD in the setting of IBD remains controversial. Historically, the literature reports widely variable rates of progression to $H G D$, ranging from $0 \%$ to $53 \% .^{10}$ Studies performed in the era of chromoendoscopy show that most patients with LGD do not progress to HGD over 3 to 4 years of follow-up.

Areas of LGD that are nonpolypoid, endoscopically invisible, $1 \mathrm{~cm}$ or larger, or preceded by indefinite dysplasia are at increased risk for progression and should be considered for colectomy. ${ }^{20}$ Other considerations for colectomy include metachronous LGD after endoscopic removal and multifocal disease. ${ }^{20}$

\section{High-Grade Dysplasia}

In HGD the risk of harboring an underlying invasive malignancy can be greater than $40 \% .{ }^{49}$ Therefore, HGD has traditionally warranted surgical resection. However, there are emerging data suggesting that discrete areas of HGD can be safely removed via endoscopy, avoiding major surgery. ${ }^{50,51}$ In 2015, the Surveillance for Colorectal Endoscopic Neoplasia Detection and Management in Inflammatory Bowel Disease Patients: International Consensus Recommendations (SCENIC) panel stated that, if dysplasia is not detected on follow-up, a decision regarding surveillance or colectomy can be individualized. ${ }^{52}$ This statement is in contrast with the European Crohn's and Colitis Organization and The American Society for Gastrointestinal Endoscopy, which state that HGD without an associated endoscopically visible lesion is an indication for surgery. ${ }^{21}$ 


\section{Strictures}

Colonic strictures develop in $5 \%$ to $10 \%$ of patients with IBD and should be routinely biopsied because of an increased risk of CRC (hazard ratio, 18.8). ${ }^{23,53,54} \mathrm{~A}$ negative biopsy may not completely rule out this risk because of sampling error and the more infiltrative nature of colitis-associated malignancies. All IBD strictures require surveillance and those that cannot be traversed and biopsied should be considered for surgical resection. ${ }^{55}$

\section{SURGICAL OPTIONS}

As with any cancer, when an IBD-CRC is found during screening or surveillance colonoscopy, preoperative staging is performed to inform appropriate treatment strategies. ${ }^{56}$

\section{Ulcerative Colitis}

In the elective setting, findings of IBD-CRC, a nonadenoma DALM, or HGD are almost uniformly accepted as indications for proctocolectomy with or without ileal pouch anal anastomosis (IPAA), ${ }^{23}$ because $43 \%$ to $50 \%$ have associated malignancy at the time of colectomy. $49,57,58$ The technical aspects of surgery for IBD-CRC mirror the approach to sporadic disease, and include high ligation of major colonic vessels and total mesorectal excision. ${ }^{59}$

In select cases, the rectum can be spared, and an ileorectal anastomosis can be performed. Although this surgery is usually less extensive, the risk of anastomotic leak is up to $13 \%$ in some studies. ${ }^{60}$ A further issue is the cumulative risk of developing dysplasia in the retained rectum $(5,10,15,20$ years is $7 \%, 9 \%, 20 \%, 25 \%$ respectively) and cancer $\left(5,10,15,20\right.$ years is $0 \%, 2 \%, 5 \%, 14 \%$ respectively). ${ }^{61,62}$

Segmental resection also has a limited role in UC because of the risk of developing secondary malignancies in other areas of colon. ${ }^{10}$ Previous reports have shown that $12 \%$ to $55 \%$ of patients have an occult or synchronous cancer and $48 \%$ have synchronous dysplasia. ${ }^{10,63,64}$

\section{Ileal Pouch Anal Anastomosis in Inflammatory Bowel Disease Colorectal Cancer}

In 1978, Parks and Nicholls ${ }^{65}$ published the technique of ileoanal pouch surgery in the British Medical Journal. ${ }^{66}$ This technique is usually accomplished in either a 2-stage or 3-stage procedure. The ultimate result with IPAA involves forming a J-shaped pouch from the terminal ileum in order to replace the function of the rectum. The presence of UC-related dysplasia or cancer is not a contraindication to IPAA.

The most common method of creating an IPAA is a double-stapled technique with a distal rectal anastomosis, preserving the anal transition zone (ATZ) ${ }^{66}$ (Fig. 1). This method leaves a cuff of residual columnar mucosa at the ATZ, which theoretically has the potential risk of future malignancy. The alternative method of IPAA creation involves performing a mucosectomy of the anal canal and a hand-sewn ileoanal anastomosis (Fig. 2). A double-stapled technique has better functional outcomes compared with a hand-sewn IPAA ${ }^{67} ; 2$ randomized trials have shown reduced levels of nocturnal fecal incontinence with the double-stapled technique. Furthermore, occasional or frequent episodes of incontinence have been reported in $64 \%$ of hand-sewn groups compared with $38 \%$ of the stapled groups. ${ }^{67-69}$

With either technique, there is a small risk of subsequent dysplasia. A 2003 study of 289 patients undergoing a double-stapled IPAA found a $4.5 \%$ incidence of dysplasia in the residual ATZ at 10 years. ${ }^{70}$ One 2011 review showed 43 known cases of dysplasia and cancer, including 30 patients with mucosectomy and 13 with a stapled 
A

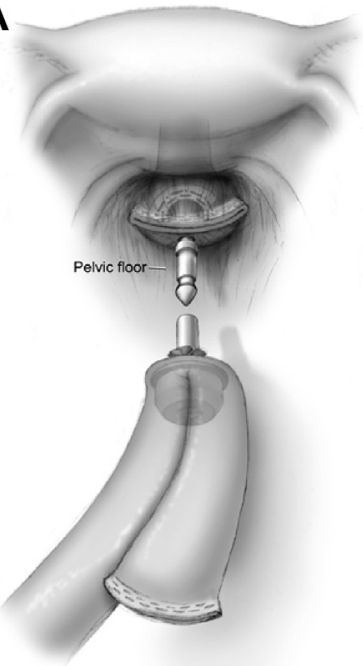

B

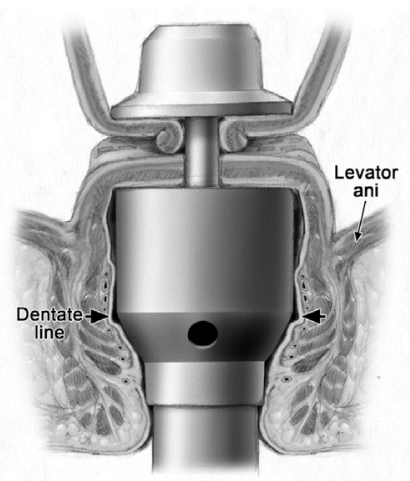

Fig. 1. Double-stapled technique for IPAA creation. $(A)$ Intraabdominal view; $(B)$ coronal anal of view IPAA creation with circular stapler. (Used with permission of Mayo Foundation for Medical Education and Research, all rights reserved.)

A

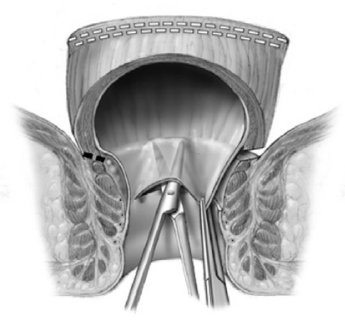

C

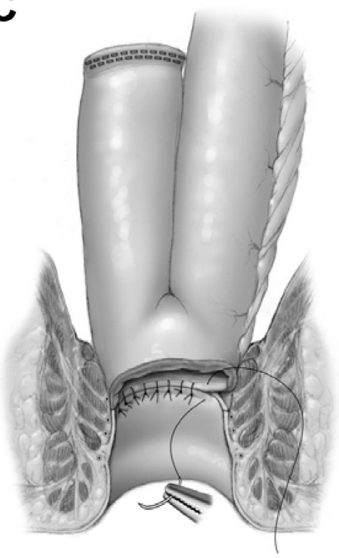

B

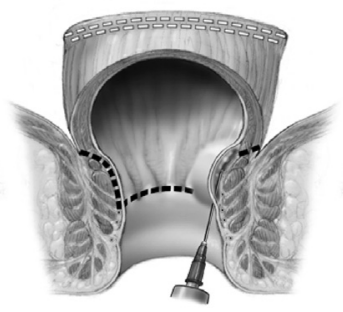

D

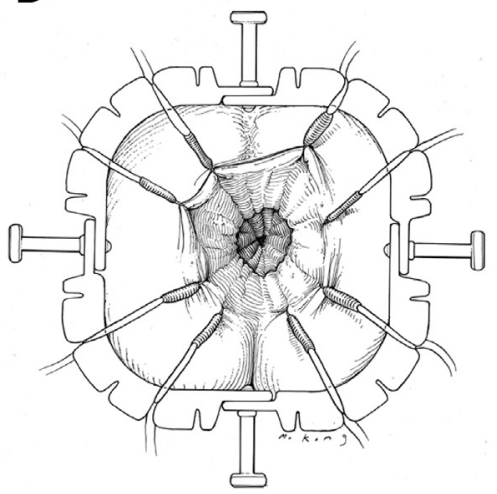

Fig. 2. Mucosectomy and hand-sewn ileoanal anastomosis. (A) Raising submucosal plain; $(B)$ excision of mucosa; $(C)$ suturing of pouch-anal anastomosis; $(D)$ external view of hand-sewn pouch-anal anastomosis. (Used with permission of Mayo Foundation for Medical Education and Research, all rights reserved.) 
anastomosis. ${ }^{71}$ Another study of 1200 patients with IBD-related IPAA found that $1.83 \%$ developed pouch neoplasia, including 16 adenocarcinomas. ${ }^{72}$ A similar review from Cleveland Clinic of 3202 patients reported that the cumulative incidences for pouch neoplasia at $5,10,15$, and 20 years were $0.9 \%, 1.3 \%, 1.9 \%$, and $4.2 \% . .^{10,73}$ Eleven $(0.36 \%)$ patients developed adenocarcinoma of the anal canal or pouch. ${ }^{73}$ The only independent risk factor for pouch neoplasia was a history of dysplasia or CRC before IPAA. ${ }^{73}$

\section{Ileal Pouch Anal Anastomosis in Inflammatory Bowel Disease Colorectal Cancer and Radiation}

Special consideration should be taken for patients with UC with locally advanced rectal cancer. Preoperative pelvic radiation can affect pouch-related sepsis and long-term pouch function. ${ }^{74} \mathrm{Wu}$ and colleagues $^{74}$ studied 63 IPAA patients with IBD-CRC and found that preoperative radiation was associated with chronic pouchitis. In the same study, pouch failure occurred in 13 patients after a median follow-up of 66.4 months. ${ }^{74}$ The investigators showed a strong association between preoperative pelvic radiation and the risk for pouch failure $(P<.001)$, but there was no correlation between pelvic radiation and pouch/anal transitional zone neoplasia, pouch stricture, pelvic abscess, and pouch fistula/sinus. ${ }^{74}$

Postoperative pelvic radiation after IPAA is even more significant and rarely allows acceptable pouch function. In 2012, Merchea and colleagues ${ }^{75}$ published on 41 patients with UC and rectal cancer, which included 11 patients undergoing IPAA. Two patients had a failed pouch, 1 of which was secondary to radiation enteritis. ${ }^{75}$ The conclusion of this study was that rectal cancer not requiring neoadjuvant chemoradiotherapy can safely undergo restorative proctocolectomy with good functional results. ${ }^{75}$ Patients who require pelvic radiation or who have stage IV disease may be better served with proctocolectomy and end ileostomy. ${ }^{75}$

\section{Crohn's Disease}

Patients with CD and HGD, multifocal LGD, a nonadenoma DALM, or invasive cancer should undergo total proctocolectomy. ${ }^{76}$ Approximately $40 \%$ of patients with CD undergoing segmental resection or subtotal colectomy develop metachronous cancers. ${ }^{76}$ Approximately $44 \%$ of patients with known malignancy have multifocal disease in the final specimen and $40 \%$ have evidence of dysplasia remote from the cancer site. ${ }^{76}$ In general, IPAA formation in the setting of CD is not recommended because it is associated with poor function and high failure rates. In highly selected patients who are not willing to have a permanent end ileostomy and have so-called rectal sparing with no active inflammation or dysplasia within the rectum, a total abdominal colectomy with ileorectal anastomosis can be considered as long as there is intense postoperative surveillance.

\section{LONG-TERM OUTCOMES}

As with sporadic CRC, in IBD-CRC the prognosis and survival depend on stage of disease and response to treatment. A study from the Mayo Clinic in 2006 comparing IBDCRC $(n=290)$ and sporadic CRC $(n=290)$ over 20 years showed comparable 5-year survival (54\% in IBD-CRC and 53\% in sporadic CRC). ${ }^{10,76}$ Several more recently published articles have also confirmed that IBD-associated CRCs have comparable rates of tumor recurrence and survival following postoperative chemotherapy with patients with CRC without IBD. ${ }^{77}$ For metastatic disease, the situation may be different. Recent work from Memorial Sloan Kettering Cancer Center case matched 18 patients with 
metastatic IBD-CRC with sporadic cases. Median overall survival was significantly worse in the IBD-related group (15 months vs 34 months). ${ }^{78}$

\section{SUMMARY}

Patients with IBD are at an increased risk of cancer secondary to long-standing intestinal inflammation. Surgical options must take into account the significant risk of synchronous disease at other colonic sites. IPAA is a viable option for patients with UC but this should be restricted to early cancers that are unlikely to require preoperative or postoperative radiation treatment.

\section{REFERENCES}

1. Dulai PS, Sandborn WJ, Gupta S. Colorectal Cancer and Dysplasia in Inflammatory Bowel Disease: A Review of Disease Epidemiology, Pathophysiology, and Management. Cancer Prev Res (Phila) 2016;9(12):887-94.

2. Crohn BB, Rosenberg B. The sigmoidoscopic picture of chronic ulcerative colitis (nonspecific). Am J Med Sci 1925;170:220-7.

3. Eaden JA, Abrams KR, Mayberry JF. The risk of colorectal cancer in ulcerative colitis: a meta-analysis. Gut 2001;48:526-35.

4. Wang ZH, Fang JY. Colorectal Cancer in Inflammatory Bowel Disease: Epidemiology, Pathogenesis and Surveillance. Gastrointest Tumors 2014;1(3):146-54.

5. Castaño-Milla C, Chaparro M, Gisbert JP. Systematic review with meta-analysis: the declining risk of colorectal cancer in UC. Aliment Pharmacol Ther 2014;39(7): $1001-2$.

6. Canavan C, Abrams KR, Mayberry J. Meta-analysis: colorectal and small bowel cancer risk in patients with Crohn's disease. Aliment Pharmacol Ther 2006;23: 1097-104.

7. Grivennikov SI. Inflammation and colorectal cancer: colitis-associated neoplasia. Semin Immunopathol 2012;35(2):229-44.

8. Fearon ER, Vogelstein B. A genetic model for colorectal Tumorigenesis. Cell 1990;61(5):759-67.

9. Munkholm P. Review article: the incidence and prevalence of colorectal cancer in inflammatory bowel disease. Aliment Pharmacol Ther 2003;18(Suppl 2):1-5.

10. Althumairi AA, Lazarev MG, Gearhart SL. Inflammatory bowel disease associated neoplasia: a surgeon's perspective. World J Gastroenterol 2016;22(3):961-73.

11. Kim ER, Chang DK. Colorectal cancer in inflammatory bowel disease: the risk, pathogenesis, prevention and diagnosis. World J Gastroenterol 2014;20(29): 9872-81.

12. Mattar MC, Lough D, Pishvaian MJ, et al. Current management of inflammatory bowel disease and colorectal cancer. Gastrointest Cancer Res 2011;4(2):53-61.

13. Andersen NN, Jess T. Has the risk of colorectal cancer in inflammatory bowel disease decreased? World J Gastroenterol 2013;19(43):7561-8.

14. Askling J, Dickman PW, Karlén P, et al. Family history as a risk factor for colorectal cancer in inflammatory bowel disease. Gastroenterology 2001;120(6):1356-62.

15. Huang LC, Merchea A. Dysplasia and Cancer in Inflammatory Bowel Disease. Surg Clin North Am 2017;97(3):627-39.

16. Mpofu C, Watson AJM, Rhodes JM. Strategies for detecting colon cancer and/or dysplasia in patients with inflammatory bowel disease. Cochrane Database Syst Rev 2004;(2). John Wiley \& Sons, Ltd, CD000279.

17. Guagnozzi D, Lucendo AJ. Colorectal cancer surveillance in patients with inflammatory bowel disease: What is new? World J Gastrointest Endosc 2012;4(4):108-16. 
18. Itzkowitz SH, Present DH. Consensus conference: Colorectal cancer screening and surveillance in inflammatory bowel disease. Inflamm Bowel Dis 2005;11:314-21.

19. Bae SI, Kim YS. Colon cancer screening and surveillance in inflammatory bowel disease. Clin Endosc 2014;47(6):509-15.

20. Choi $\mathrm{CH}$, Ignjatovic-Wilson A, Askari A, et al. Low-grade dysplasia in ulcerative colitis: risk factors for developing high-grade dysplasia or colorectal cancer. Am J Gastroenterol 2015;110(10):1461-71.

21. Huguet JM, Suárez P, Ferrer-Barceló L, et al. Endoscopic recommendations for colorectal cancer screening and surveillance in patients with inflammatory bowel disease: Review of general recommendations. World J Gastrointest Endosc 2017;9(6):255-62.

22. Broomé U, Löfberg R, Veress B, et al. Primary sclerosing cholangitis and ulcerative colitis: evidence for increased neoplastic potential. Hepatology 1995;22:1404-8.

23. Ross H, Steele SR, Varma M, et al. Practice Parameters for the Surgical Treatment of Ulcerative Colitis. Dis Colon Rectum 2014;57:5-22.

24. Awais D, Siegel CA, Higgins PD. Modelling dysplasia detection in ulcerative colitis: clinical implications of surveillance intensity. Gut 2009;58:1498-503.

25. Rubin CE, Haggitt RC, Burmer GC, et al. DNA aneuploidy in colonic biopsies predicts future development of dysplasia in ulcerative colitis. Gastroenterology 1992; 103:1611-20.

26. Blonski W, Kundu R, Lewis J, et al. Is dysplasia visible during surveillance colonoscopy in patients with ulcerative colitis? Scand J Gastroenterol 2008;43: 698-703.

27. Rubin DT, Rothe JA, Hetzel JT, et al. Are dysplasia and colorectal cancer endoscopically visible in patients with ulcerative colitis? Gastrointest Endosc 2007;65: 998-1004.

28. Watanabe T, Ajioka Y, Mitsuyama K, et al. Comparison of Targeted vs Random Biopsies for Surveillance of Ulcerative Colitis-Associated Colorectal Cancer. Gastroenterology 2016;151:1122-30.

29. Rutter MD, Saunders BP, Wilkinson KH, et al. Most dysplasia in ulcerative colitis is visible at colonoscopy. Gastrointest Endosc 2004;6:334-9.

30. Trivedi PJ, Braden B. Indications, stains and techniques in chromoendoscopy. QJM 2012;106(2):117-31.

31. Barkin JA, Sussman DA, Abreu MT. Chromoendoscopy and advanced imaging technologies for surveillance of patients with IBD. Gastroenterol Hepatol (N Y) 2012;8(12):796-802.

32. Subramanian V, Mannath J, Ragunath K, et al. Meta-analysis: the diagnostic yield of chromoendoscopy for detecting dysplasia in patients with colonic inflammatory bowel disease. Aliment Pharmacol Ther 2011;33(3):304-12.

33. Shukla R, Salem M, Hou JK. Use and barriers to chromoendoscopy for dysplasia surveillance in inflammatory bowel disease. World J Gastrointest Endosc 2017; 9(8):359-67.

34. Cho WY, Jang JY, Lee DH, Endoscopic Technology and Investigation Study Group. Recent Advances in Image-enhanced Endoscopy. Clin Endosc 2011; 44(2):65-75.

35. Pellisé M, López-Cerón M, Rodríguez de Miguel C, et al. Narrow-band imaging as an alternative to chromoendoscopy for the detection of dysplasia in longstanding inflammatory bowel disease: a prospective, randomized, crossover study. Gastrointest Endosc 2011;74(4):840-8. 
36. Dekker E, van den Broek FJ, Reitsma JB, et al. Narrow-band imaging compared with conventional colonoscopy for the detection of dysplasia in patients with longstanding ulcerative colitis. Endoscopy 2007;39:216-21.

37. Galli J, Cammarota G, Rigante M, et al. High resolution magnifying endoscopy: a new diagnostic tool also for laryngeal examination? Acta Otorhinolaryngol Ital 2007;27(5):233-6.

38. Kiesslich R, Goetz M, Neurath MF. Confocal laser endomicroscopy for gastrointestinal diseases. Gastrointest Endosc Clin N Am 2008;18:451-66.

39. Kim ES. Role of Advanced Endoscopic Imaging Techniques in the Management of Inflammatory Bowel Disease. Clin Endosc 2017;50(5):424-8.

40. Beintaris I, Rutter M. Advanced imaging in colonoscopy: contemporary approach to dysplasia surveillance in inflammatory bowel disease. Frontline Gastroenterol 2016;7(4):308-15.

41. Günther U, Kusch D, Heller F, et al. Surveillance colonoscopy in patients with inflammatory bowel disease: comparison of random biopsy vs. targeted biopsy protocols. Int J Colorectal Dis 2011;26:667-72.

42. Freire P, Figueiredo $P$, Cardoso R. Surveillance in ulcerative colitis: is chromoendoscopy-guided endomicroscopy always better than conventional coIonoscopy? A randomized trial. Inflamm Bowel Dis 2014;20:2038-45.

43. Wanders LK, Kuiper T, Kiesslich R. Limited applicability of chromoendoscopyguided confocal laser endomicroscopy as daily-practice surveillance strategy in Crohn's disease. Gastrointest Endosc 2016;83:966-71.

44. Harpaz N, Polydorides AD. Colorectal Dysplasia in IBD. Arch Pathol Lab Med 2010;134:876-95.

45. Fogt F, Urbanski SJ, Sanders ME, et al. Distinction between dysplasia-associated lesion or mass (DALM) and adenoma in patients with ulcerative colitis. Hum Pathol 2000;31(3):288-91.

46. Quinn AM, Farraye FA, Naini BV, et al. Polypectomy is adequate treatment for adenoma-like dysplastic lesions (DALMs) in Crohn's disease. Inflamm Bowel Dis 2013;19(6):1186-93.

47. Wanders LK, Dekker E, Pullens B, et al. Cancer risk after resection of polypoid dysplasia in patients with longstanding ulcerative colitis: A Meta-analysis. Clin Gastroenterol Hepatol 2014;12:756-64.

48. Thomas T, Abrams KA, Robinson RJ, et al. Meta-analysis: cancer risk of lowgrade dysplasia in chronic ulcerative colitis. Aliment Pharmacol Ther 2007;25: 657-68.

49. Bernstein CN, Shanahan F, Weinstein WM. Are we telling patients the truth about surveillance colonoscopy in ulcerative colitis? Lancet 1994;343:71-4.

50. Blonski W, Kundu R, Furth EF, et al. High-grade dysplastic adenoma-like mass lesions are not an indication for colectomy in patients with ulcerative colitis. Scand J Gastroenterol 2008;43:817.

51. Smith LA, Baraza W, Tiffin N, et al. Endoscopic resection of adenoma-like mass in chronic ulcerative colitis using a combined endoscopic mucosal resection and cap assisted submucosal dissection technique. Inflamm Bowel Dis 2008;14: 1380.

52. Laine L, Kaltenbach T, Barkun A, et al. SCENIC international consensus statement on surveillance and management of dysplasia in inflammatory bowel disease. Gastroenterology 2015;148(3):639-51.

53. Lashner BA, Turner BC, Bostwick DG, et al. Dysplasia and cancer complicating strictures in ulcerative coli- tis. Dig Dis Sci 1990;35:349-52. 
54. Lovasz BD, Lakatos L, Golovics PA, et al. Risk of Colorectal Cancer in Crohn s Disease Patients with Colonic Involvement and Stenosing Disease in a Population-based Cohort from Hungary. J Gastrointestin Liver Dis 2013;22(3): 265-8.

55. Hwang JM, Varma MG. Surgery for inflammatory bowel disease. World J Gastroenterol 2008;14(17):2678-90.

56. Wu JS. Rectal cancer staging. Clin Colon Rectal Surg 2007;20(3):148-57.

57. Blackstone $\mathrm{MO}$, Riddell $\mathrm{RH}$, Rogers $\mathrm{BH}$, et al. Dysplasia- associated lesion or mass (Dalm) detected by colonoscopy in long-standing ulcerative colitis: an indication for colectomy. Gastroenterology 1981;80:366-74.

58. Riddell RH, Goldman H, Ransohoff DF. Dysplasia in inflammatory bowel disease: standardized classification with pro- visional clinical applications. Hum Pathol $1983 ; 14: 931-68$.

59. Yao HW, Liu YH. Re-examination of the standardization of colon cancer surgery. Gastroenterol Rep (Oxf) 2013;1(2):113-8.

60. Segelman J, Mattsson I, Jung B, et al. Risk factors for anastomotic leakage following ileosigmoid or ileorectal anastomosis. Colorectal Dis 2018;20(4): 304-11.

61. Juviler A, Hyman N. Ulcerative colitis: the fate of the retained rectum. Clin Colon Rectal Surg 2004;17(1):29-34.

62. Scoglio D, Ahmed Ali U, Fichera A. Surgical treatment of ulcerative colitis: ileorectal vs ileal pouch-anal anastomosis. World J Gastroenterol 2014;20(37): 13211-8.

63. Beaugerie L, Svrcek M, Seksik P, et al. Risk of colorectal high-grade dysplasia and cancer in a prospective observational cohort of patients with inflammatory bowel disease. Gastroenterology 2013;145:166-75.

64. Jayaram H, Satsangi J, Chapman RW. Increased colorectal neoplasia in chronic ulcerative colitis complicated by primary sclerosing cholangitis: fact or fiction? Gut 2001;48:430-4.

65. Parks AG, Nicholls RJ. Proctocolectomy without ileostomy for ulcerative colitis. BMJ 1978;2:85-8.

66. Carne PW, Pemberton JH. Technical aspects of ileoanal pouch surgery. Clin CoIon Rectal Surg 2004;17(1):35-41.

67. Deen KI, Williams JG, Grant EA, et al. Randomized trial to determine the optimum level of pouch-anal anastomosis in stapled restorative proctocolectomy. Dis CoIon Rectum 1995;38(2):133-8.

68. Reilly WT, Pemberton JH, Wolff BG, et al. Randomized prospective trial comparing ileal pouch-anal anastomosis performed by excising the anal mucosa to ileal pouch-anal anastomosis performed by preserving the anal mucosa. Ann Surg 1997;225(6):666-76.

69. Hallgren TA, Fasth SB, Oresland TO, et al. Ileal pouch anal function after endoanal mucosectomy and handsewn ileoanal anastomosis compared with stapled anastomosis without mucosectomy. Eur J Surg 1995;161(12):915-21.

70. Remzi FH, Fazio VW, Delaney CP, et al. Dysplasia of the anal transitional zone after ileal pouch-anal anastomosis: results of prospective evaluation after a minimum of ten years. Dis Colon Rectum 2003;46(1):6-13.

71. Um JW, M'Koma AE. Pouch-related dysplasia and adenocarcinoma following restorative proctocolectomy for ulcerative colitis. Tech Coloproctol 2011; 15(1):7-16. 
72. Derikx LA, Kievit W, Drenth JP, et al. Prior colorectal neoplasia is associated with increased risk of ileoanal pouch neoplasia in patients with inflammatory bowel disease. Gastroenterology 2014;146(1):119-28.

73. Kariv R, Remzi FH, Lian L, et al. Preoperative colorectal neoplasia increases risk for pouch neoplasia in patients with restorative proctocolectomy. Gastroenterology 2010;139:806-12.

74. Wu XR, Kiran RP, Remzi FH, et al. Preoperative pelvic radiation increases the risk for ileal pouch failure in patients with colitis-associated colorectal cancer. J Crohns Colitis 2013;7(10):e419-26.

75. Merchea A, Wolff BG, Dozois EJ, et al. Clinical features and oncologic outcomes in patients with rectal cancer and ulcerative colitis: a single-institution experience. Dis Colon Rectum 2012;55(8):881-5.

76. Delaunoit T, Limburg PJ, Goldberg RM, et al. Colorectal cancer prognosis among patients with inflammatory bowel disease. Clin Gastroenterol Hepatol 2006;4: 335-42.

77. Dugum M, Lin J, Lopez R, et al. Recurrence and survival rates of inflammatory bowel disease-associated colorectal cancer following postoperative chemotherapy: a comparative study. Gastroenterol Rep (Oxf) 2016;5(1):57-61.

78. Yaeger, Hersch J, Bates D, et al. Outcomes for matched patients with metastatic colitis-associated cancers versus sporadic colorectal cancer receiving chemotherapy. J Clin Oncol 2018;36(15S):e15534. 